\title{
Normalizability of One-dimensional Quasi-exactly Solvable Schrödinger Operators
}

\author{
Artemio González-López ${ }^{1 \star}$, Niky Kamran ${ }^{2 \star \star}$ and Peter J. Olver ${ }^{3 \star \star \star, \star \star \star \star \star}$ \\ 1 Departamento de Física Teórica II, Universidad Complutense, 28040 Madrid, Spain \\ 2 Department of Mathematics, McGill University, Montréal, Québec, Canada H3A 2K6 \\ ${ }^{3}$ Department of Mathematics, University of Maryland, College Park, MD 20742, U.S.A.
}

Received July 7, 1992

\begin{abstract}
We completely determine necessary and sufficient conditions for the normalizability of the wave functions giving the algebraic part of the spectrum of a quasi-exactly solvable Schrödinger operator on the line. Methods from classical invariant theory are employed to provide a complete list of canonical forms for normalizable quasi-exactly solvable Hamiltonians and explicit normalizability conditions in general coordinate systems.
\end{abstract}

\section{Introduction}

Lie algebraic and Lie group theoretic methods have played a significant role in the development of quantum mechanics since its inception. In the classical applications, the Lie group appears as a symmetry group of the Hamiltonian operator, and the associated representation theory provides an algebraic means for computing the spectrum. Of particular importance are the exactly solvable problems, such as the harmonic oscillator or the hydrogen atom, whose point spectrum can be completely determined using purely algebraic methods. In the early 1980's, in order to study molecular spectroscopy, Alhassid, Gürsey, Iachello, Levine, and collaborators, [2, 3, 1, 14], introduced the concept of a "spectrum generating algebra" to construct models for complicated molecules whose point spectrum could be analyzed algebraically. The Schrödinger operators amenable to the algebraic approach to scattering assumed a "Lie algebraic form," meaning that they belong to the universal enveloping algebra of the spectrum generating algebra. Thus, a second order differential operator

\footnotetext{
* Supported in Part by DGICYT Grant PS 89-0011

$\star \star$ Supported in Part by an NSERC Grant

$\star \star \star$ Supported in Part by NSF Grant DMS 92-04192

$\star \star \star \star$ On leave from School of Mathematics, University of Minnesota, Minneapolis, Minnesota 55455, U.S.A
} 
(Hamiltonian) $\mathscr{H}$ is said to be in Lie algebraic form if it can be written as a quadratic combination

$$
\mathscr{H}=\sum_{a, b} c_{a b} J^{a} J^{b}+\sum_{a} c_{a} J^{a}
$$

of a collection of first order differential operators $J^{a}$ which generate a finitedimensional Lie algebra $\mathfrak{g}=\operatorname{Span}\left\{J^{a}\right\}$. (In our Lie algebraic form (1.1), we could also include a constant term if desired, although this merely has the effect of translating the spectrum.) Note that if $J^{c}$ is any generator of the Lie algebra $\mathfrak{g}$, its commutator $\left[J^{c}, \mathscr{H}\right]$ with the Hamiltonian operator, while still of Lie algebraic form, is not in general a multiple of $\mathscr{H}$. (However, if $\mathscr{H}$ is a Casimir for the Lie algebra, then it is invariant in the usual sense.) Thus the Lie algebra $g$ is not a symmetry algebra in the traditional sense, but is referred to as a "hidden symmetry algebra" of the operator (1.1). Lie algebraic operators reappeared in the remarkable discovery of Turbiner, Shifman, Ushervidze, and their collaborators, $[22,19,21,24]$, of a new class of physically significant spectral problems, which they named "quasi-exactly solvable problems," having the property that a (finite) part of the point spectrum can be determined using purely algebraic methods. Any quasi-exactly solvable Schrödinger operator will be in the general Lie algebraic form (1.1), but the Lie algebra $g$ must satisfy the additional condition that it possesses a finite-dimensional representation space $\mathscr{N}$ consisting of smooth wave functions. In this case, the Hamiltonian (1.1) clearly restricts to a linear transformation on the finite-dimensional space $\mathcal{N}$, and hence the associated eigenvalues can be computed by purely algebraic methods, i.e., matrix eigenvalue calculations. Additional impetus for the study of such problems stems from the analogy between the Lie algebraic form (1.1) of a quasi-exactly solvable Hamiltonian and the generalized Sugawara construction of the stress-energy tensor in two-dimensional conformal field theory noted in [17]. Subsequently, Gorsky, [8], discovered a direct relation between one-dimensional quasi-exactly solvable problems and threeand four-point conformal blocks in a conformal field theory with a zero vector at the second level; see also the recent review paper by Shifman, [20].

For one-dimensional problems, the complete classification of (second order) Lie algebraic and quasi-exactly solvable Schrödinger operators is fairly elementary for two reasons. Firstly, there is essentially just a single parametrized family of finite-dimensional Lie algebras of first order differential operators. Secondly, every second order differential operator is equivalent to a Schrödinger operator $\mathscr{S}=-D_{x}^{2}+V(x)$, with potential $V(x)$. Complete lists of all one-dimensional Lie algebraic potentials appear in [12], and all quasi-exactly solvable potentials in [22]; they include many of the well-known potentials from basic quantum mechanics, such as the (radial) harmonic oscillator, one-soliton (Pöschl-Teller), Morse, and elliptic function potentials, as well as several new potentials of interest. Most of the more recent work has, therefore, concentrated on higher dimensional problems. See $[5,6]$, for complete classifications of all finite-dimensional Lie algebras of first order differential operators in two (complex) variables and their quasi-exactly solvable versions. See [21] for examples of planar quasi-exactly solvable potentials and [7] for a more detailed, but still far from complete, treatment of twodimensional problems.

There is, however, one important question for one-dimensional problems which has not been previously addressed in the literature, and whose solution is the main 
result of this paper. Namely, while one can determine a number of eigenvalues of any quasi-exactly solvable Schrödinger operator (1.1), it is not immediately clear whether, in the physical coordinates, the associated eigenfunctions are actually normalizable, meaning that they lie in the Hilbert space $\mathrm{L}^{2}$, and so represent true physical wave functions. Or, stated another way, which of the algebraically computed eigenvalues of a quasi-exactly solvable Schrödinger operator represent genuine physical bound states? One is interested in conditions on the potential $V(x)$, or, perhaps more importantly in view of the previously mentioned connections with conformal field theory, on the coefficients $c_{a b}, c_{a}$, in the Lie algebraic form (1.1) of the operator. In this paper, we deduce a complete set of explicit necessary and sufficient conditions, both on the potential and on the coefficients, for the normalizability of the eigenfunctions of a general second order quasi-exactly solvable Schrödinger operator. A solution to the normalizability problem, when combined with very recent work of Turbiner, [23], has direct applications to the "Bochner problem," [15], which is to characterize differential operators possessing orthogonal polynomial eigenfunctions.

Our solution to the normalizability problem relies on an unusual combination of direct asymptotic methods and techniques from classical invariant theory, $[9,11]$. We first prove that any one-dimensional quasi-exactly solvable Schrödinger operator is uniquely determined by two polynomials, one, $P(z)$, of degree four (a "quartic") and the second, $Q(z)$, of degree two (a "quadratic"), and a single constant; the latter plays an inessential role, and can be absorbed by merely translating the spectrum of the operator. The group $\operatorname{GL}(2, \mathbb{R})$ of linear fractional transformations on the line acts on these polynomials according to the standard representations of weights $(4,-2)$ and $(2,-1)$, respectively, and hence can be used to place the operator into a simple canonical form. For an operator in canonical form, the explicit change of variables required to place it in physical Schrödinger form is readily constructed, as are the formulas for the potential and the eigenfunctions. Consequently, the normalizability conditions for the canonical form, and hence the potential itself, are readily found by direct inspection. It should also be remarked that our classification yields a complete list of exactly solvable potentials with normalizable eigenfunctions which includes several new cases not noted previously.

Translation of the conditions for normalizability into conditions on the coefficients of the Lie algebraic form (1.1) rests on the following key observation. For a given quasi-exactly solvable Schrödinger operator, the condition that its eigenfunctions be normalizable in the physical variables is invariant under the action of the linear fractional transformation group $\mathrm{GL}(2, \mathbb{R})$. Moreover, any property of a quasi-exactly solvable Schrödinger operator which is invariant under the action of $\operatorname{GL}(2, \mathbb{R})$ will also be an invariant property of the two polynomials $P, Q$, which determine the operator, and can therefore be expressed in terms of the classical joint invariants and covariants of the two polynomials. The covariants, in turn, can be readily re-expressed in terms of the Lie algebraic coefficients $c_{a b}, c_{a}$. Therefore, we need to reformulate our explicit normalizability conditions for the canonical form of the operator in terms of the joint covariants of the pair of polynomials $P, Q$, in order to find the required conditions on the Lie algebraic coefficients. The requisite covariants themselves are obtained through the invariant-theoretic process of "transvection," and, moreover, a complete system (a "Hilbert basis") of covariants of a quartic and a quadratic is known, so the invariant version of the normalizability conditions can be found by inspection. For example, the simplest 
condition for normalizability is that the quartic polynomial have one (or more) multiple roots, and is expressed invariantly by the vanishing of its discriminant; the resulting condition is an explicit sixth degree polynomial constraint on the coefficients, cf. (6.9) below. Although most of the normalizability conditions are, unfortunately, quite complicated, they will be exhibited in (semi-)explicit form in the final section of the paper.

\section{Quasi-exactly Solvable Schrödinger Operators on the Line}

Let us begin by reviewing the known theory of one-dimensional quasi-exactly solvable spectral problems. First, for classifying (Lie algebras of) differential operators, we need to specify the allowable changes of variables. In our case, two differential operators are equivalent if they can be mapped into each other by a combination of change of independent variable, $x=\xi(z)$, and "gauge transformation" $e^{\alpha(x)} \cdot \mathscr{H} \cdot e^{-\alpha(x)}$. The gauge factor $\mu(x)=e^{\alpha(x)}$ is not necessarily unitary, i.e., $\alpha(x)$ is not restricted to be purely imaginary, and hence does not preserve the normalizability properties of the associated eigenfunctions. Note that the changes of variables and gauge transformations both respect the commutator $[\mathscr{H}, \mathscr{D}]$ between differential operators, and hence preserve the Lie algebra structure.

A classical elementary result (which is not valid in higher dimensions) states that any second order differential operator is equivalent, under such transformations, to a Schrödinger operator, cf. [25].

Proposition 1. Let $\mathscr{H}=-P(z) D_{z}^{2}-Q(z) D_{z}-R(z)$ be a second order differential operator such that $P(z)>0$. Then there is a (local) change of variables $x=\xi(z)$ and nonzero gauge factor $\mu(x)$ which transforms $\mathscr{H}$ to a Schrödinger operator $\mathscr{S}=-D_{x}^{2}+V(x)$. The potential $V(x)$ is uniquely determined up to translation $V(x) \mapsto V(x+\delta)$.

The minus signs in the formula for $\mathscr{H}$ are for later convenience. See Theorem 7 below for a more detailed version of this result.

Here we are interested in determining whether a given second order differential operator can be written in the Lie algebraic form (1.1). Two Lie algebras of differential operators are equivalent if they can be mapped to each other by a change of variables and gauge transformation. There is a complete classification of (generic) Lie algebras of first order differential operators in one (and two) dimensions, $[12,5]$. The additional condition that the Lie algebra be quasi-exactly solvable, meaning that it has a finite-dimensional module (representation space) of smooth functions has also been thoroughly analyzed in both cases, $[22,6]$. Here we state the one-dimensional classification.

Theorem 2. Every (generic) finite-dimensional quasi-exactly solvable Lie algebra of first order differential operators in one (real or complex) variable is, locally, equivalent to a subalgebra of one of the Lie algebras

$$
\mathfrak{g}_{n}=\operatorname{Span}\left\{D_{z}, z D_{z}, z^{2} D_{z}-n z, 1\right\},
$$

where $n \in \mathbb{N}$ is a non-negative integer. The associated module $\mathscr{P}^{(n)}$ consists of the polynomials of degree at most $n$. 
We will refer to the coordinate $z$ in which the Lie algebra takes the canonical form (2.1) as the "canonical" or "gauged" coordinate, to be distinguished from the physical coordinate $x$ in which the Hamiltonian takes the form of a Schrödinger operator. See [6] for a cohomological interpretation of the parameter $n$. Physically, the half-integer $\frac{1}{2} n$ is the analogue of the "spin" of the quantum mechanical system. Note that even when $n$ is non-integral, $\mathfrak{g}_{n}$ remains a Lie algebra, but it no longer possesses a finite-dimensional module consisting of smooth functions $f(z)$. The fact that $n$ can only assume discrete values in order that the Lie algebra satisfy the quasi-exact solvability condition is a specific case of the apparently general, but as yet poorly understood, phenomenon labelled "quantization of cohomology," first noted in [6].

As an abstract Lie algebra, $\mathfrak{g}_{n}$ is merely a central extension (by the constant functions) of the subalgebra $\mathfrak{h}_{n}$ spanned by the differential operators

$$
J^{-}=J_{n}^{-}=D_{z}, \quad J^{0}=J_{n}^{0}=z D_{z}-\frac{n}{2}, \quad J^{+}=J_{n}^{+}=z^{2} D_{z}-n z,
$$

which realize the standard commutation relations on the Lie algebra $\mathfrak{s l}(2, \mathbb{R})$, and so $\mathfrak{g}_{n}$ is isomorphic to the Lie algebra $\mathrm{gl}(2)$ of all $2 \times 2$ matrices. Note that since $\mathfrak{g}_{n}$ and $\mathfrak{h}_{n}$ only differ by inclusion of constant functions, in our analysis of Lie algebraic differential operators we can, without loss of generality, concentrate on the Lie algebra $\mathfrak{h}_{n}$, since any Lie algebraic operator (1.1) for the full algebra $\mathfrak{g}_{n}$ is automatically a Lie algebraic operator for the subalgebra $\mathfrak{h}_{n}$.

As a direct consequence of Theorem 2, the most general second order quasiexactly solvable Hamiltonian in one space dimension can be written (after a gauge transformation) in the form

$$
\begin{aligned}
-\mathscr{H}= & c_{++}\left(J^{+}\right)^{2}+c_{+0}\left[J^{+} J^{0}+J^{0} J^{+}\right]+c_{00}\left(J^{0}\right)^{2} \\
& +c_{+-}\left[J^{+} J^{-}+J^{-} J^{+}\right]+c_{0-}\left[J^{0} J^{-}+J^{-} J^{0}\right]+c_{--}\left(J^{-}\right)^{2} \\
& +c_{+} J^{+}+c_{0} J^{0}+c_{-} J^{-}+c_{*} .
\end{aligned}
$$

(In general, since the $J^{a}$ span a Lie algebra, we can, without loss of generality, assume that the coefficients in (1.1) are symmetric: $c_{a b}=c_{b a}$, and so only the anti-commutators $J^{a} J^{b}+J^{b} J^{a}$ appear in the first summation.) Substituting the explicit formulas (2.2) into the expression (2.3), we see that every quasi-exactly solvable operator can be written in the canonical $z$ coordinate in the form

$$
-\mathscr{H}=P(z) D_{z}^{2}+\tilde{Q}(z) D_{z}+\tilde{R}(z),
$$

where $P, \tilde{Q}, \tilde{R}$ are polynomials

$$
\begin{aligned}
& P(z)=a z^{4}+b z^{3}+c z^{2}+d z+e, \\
& \tilde{Q}(z)=2(1-n) a z^{3}+\tilde{b} z^{2}+\tilde{c} z+\tilde{d}, \\
& \tilde{R}(z)=n(n-1) a z^{2}-n[(n-1) b+\tilde{b}] z+f .
\end{aligned}
$$

Here $a, b, c$, etc., are constants, and $n$ is the quantized parameter determing the Lie algebra. However, instead of using $P, \tilde{Q}, \tilde{R}$ as the primary polynomials of interest, we define

$$
Q(z)=\tilde{Q}(z)+\frac{n-1}{2} P^{\prime}(z), \quad R(z)=\tilde{R}(z)+\frac{n}{2} \tilde{Q}^{\prime}(z)+\frac{n(n-1)}{6} P^{\prime \prime}(z) .
$$


Then $P, Q, R$ are polynomials of respective degrees $4,2,0$, and are given explicitly as

$$
P(z)=a z^{4}+b z^{3}+c z^{2}+d z+e, \quad Q(z)=\hat{b} z^{2}+\hat{c} z+\hat{d}, \quad R(z)=c^{*},
$$

where, in terms of the coefficients in (2.3),

$$
\begin{gathered}
a=c_{++}, \quad b=2 c_{+0}, \quad c=2 c_{+-}+c_{00}, \quad d=2 c_{0-}, \quad e=c_{--}, \\
\hat{b}=c_{+}, \quad \hat{c}=c_{0}, \quad \hat{d}=c_{-}, \quad c^{*}=\frac{n^{2}+2 n}{12} c_{00}-\frac{n^{2}+2 n}{3} c_{+-}+c_{*} .
\end{gathered}
$$

Note that the final constant $c_{*}$ in $(2.3)$ is redundant. The Hamiltonian has the form

$$
-\mathscr{H}=P(z) D_{z}^{2}+\left\{Q(z)-\frac{n-1}{2} P^{\prime}(z)\right\} D_{z}+\left\{R-\frac{n}{2} Q^{\prime}(z)+\frac{n(n-1)}{12} P^{\prime \prime}(z)\right\} .
$$

Note that, by translating the spectrum of $\mathscr{H}$, we can eliminate the constant $R=c^{*}$, and so such operators are essentially prescribed by their quartic and quadratic polynomial coefficients $P(z), Q(z)$.

The associated finite-dimensional representation space (module) for the Lie algebra $\mathfrak{h}_{n}$ is the space $\mathscr{P}^{(n)}$ consisting of all polynomials $\chi(z)$ of degree at most $n$. Thus the algebraic eigenfunctions of the quasi-exactly solvable Hamiltonian (2.3) will, in the gauged $z$-coordinates, just be polynomials $\chi_{k}(z)$ of degree at most $n$, and we can compute $n$ different eigenvalues via this algebraic approach.

Note that if we introduce the standard basis $\phi_{k}(z)=z^{k}, k=0, \ldots, n$ of the representation space $\mathscr{P}^{(n)}$, then the generators $J^{+}, J^{-}$of $\mathfrak{h}_{n}$ act as ordinary raising and lowering operators:

$$
J^{-}\left(\phi_{k}\right)=k \phi_{k-1}, \quad J^{0}\left(\phi_{k}\right)=\left(k-\frac{n}{2}\right) \phi_{k}, \quad J^{+}\left(\phi_{k}\right)=(k-n) \phi_{k+1} .
$$

This immediately implies that the reduced transformation takes on a rather simple matrix form, and hence its spectrum can be computed by fairly simple linear algebraic (or numerical) methods.

Proposition 3. Let $M=\mathscr{H} \mid \mathscr{P}^{(n)}$ denote the restriction of the quasi-exactly solvable differential operator (2.3) to the representation space $\mathscr{P}^{(n)}$. Then, with respect to the standard basis $\phi_{k}(z)=z^{k}, k=0, \ldots, n$, of $\mathscr{P}^{(n)}$, the linear transformation $M$ takes the form of a pentadiagonal matrix, or, if $c_{++}=c_{--}=0$, a tridiagonal matrix.

Indeed, the matrix representation of the Hamiltonian only depends on its Lie algebraic form (2.3), and not on the particular coordinate system used to represent it as a second order differential operator. Therefore, all the algebraic eigenvalues can be computed in the simpler gauged coordinates; the only question, then, is whether these represent genuine physical spectrum of the operator, i.e., whether the eigenfunctions are, in the physical coordinates, normalizable. Our solution to the normalizability problem will allow us to answer this question. 


\section{Action of $G L(2, \mathbb{R})$ on the Hamiltonian}

The canonical form (2.9) of a quasi-exactly solvable differential operator is not unique, since there is a residual (hidden) symmetry group which preserves the quasi-exactly solvable Lie algebra $\mathfrak{h}_{n}$. Not surprisingly, this group is $\mathrm{GL}(2)=\mathrm{GL}(2, \mathbb{R})$, which acts on the (projective) line according to the linear fractional, or Möbius, transformations

$$
z \mapsto w=\frac{\alpha z+\beta}{\gamma z+\delta}, \quad A=\left(\begin{array}{cc}
\alpha & \beta \\
\gamma & \delta
\end{array}\right), \quad \operatorname{det} A=\Delta=\alpha \delta-\beta \gamma \neq 0 .
$$

There is an induced action of GL(2) on the space $\mathscr{P}^{(n)}$ of polynomials of degree at most $n$, which maps a polynomial $P(w) \in \mathscr{P}^{(n)}$ to the polynomial

$$
P(w) \mapsto \tilde{P}(z)=(\gamma z+\delta)^{n} P\left(\frac{\alpha z+\beta}{\gamma z+\delta}\right) .
$$

We let $\rho_{n, 0}$ denote this (irreducible) multiplier representation of GL(2), which is isomorphic to the standard representation on homogeneous polynomials of degree $n$ in two variables, with GL(2) acting via matrix multiplication on the variables. Indeed, the differential operators (2.2) are just the infinitesimal generators of the multiplier representation $\rho_{n, 0}$ of GL(2), [16]. Therefore, the representation $\rho_{n, 0}$ induces an automorphism of the Lie algebra $\mathfrak{h}_{n}$, and hence of the space of associated quasi-exactly solvable Hamiltonian operators. Explicitly, the action on the generators (2.2) of $\mathfrak{h}_{n}$ is

$$
\left(\begin{array}{l}
J^{-} \\
J^{0} \\
J^{+}
\end{array}\right) \mapsto \frac{1}{\Delta}\left(\begin{array}{ccc}
\delta^{2} & 2 \gamma \delta & \gamma^{2} \\
\beta \delta & \alpha \delta+\beta \gamma & \alpha \gamma \\
\beta^{2} & 2 \alpha \beta & \alpha^{2}
\end{array}\right)\left(\begin{array}{l}
J^{-} \\
J^{0} \\
J^{+}
\end{array}\right),
$$

independent of $n$, which, apart from the determinantal factor $\Delta=\operatorname{det} A$, can be identified with the standard representation $\rho_{2,0}$ of GL(2) on the space of homogeneous quadratic polynomials in two variables. More correctly, this representation is isomorphic to the tensor product representation $\rho_{2,-1}=\rho_{2,0} \otimes \operatorname{det}^{-1}=$ $\rho_{2,0} \otimes \rho_{0,-1}$ of the standard quadratic polynomial representation $\rho_{2,0}$ with the inverse of the one-dimensional determinantal representation $\rho_{0,1}: A \mapsto \operatorname{det} A$.

Definition 4. Let $n \geqq 0, i$ be integers. Define the multiplier representation $\rho_{n, i}$ of the general linear group $\mathrm{GL}(2)$ on the space $\mathscr{P}^{(n)}$ of polynomials of degree at most $n$ by

$$
P(w) \mapsto \hat{P}(z)=(\gamma z+\delta)^{n}(\alpha \delta-\beta \gamma)^{i} P\left(\frac{\alpha z+\beta}{\gamma z+\delta}\right), \quad P \in \mathscr{P}^{(n)} .
$$

Note that $\rho_{n, i}$ is isomorphic to the tensor product of the $i^{\text {th }}$ power of the determinantal representation with the standard representation on $\mathscr{P}^{(n)}$, i.e.,

$$
\rho_{n, i}=\rho_{n, 0} \otimes \operatorname{det}^{i}=\rho_{n, 0} \otimes \rho_{0, i} .
$$

Let $P(z)$ be a polynomial of degree $d \leqq n$, transforming under a representation $\rho_{n, i}$. A finite point $z_{0}$ is a root of $P$ if $P\left(z_{0}\right)=0$. We let $\mathcal{O}\left(P, z_{0}\right)=m$ denote the order of the root, so that $P(z) \sim \alpha\left(z-z_{0}\right)^{m}$ as $z \rightarrow z_{0}$. In particular, $\mathcal{O}\left(P, z_{0}\right)=0$ if $P$ does not vanish at $z_{0}$. The definition of order can be extended to $z_{0}=\infty$ by 
defining $\mathcal{O}(P, \infty)=n-d$, so that $\infty$ is a root of $P$ if and only if the degree $d$ of $P$ is strictly less than $n$, the weight of the representation. (Using this definition, we see that every non-zero polynomial belonging to the representation $\rho_{n, i}$ has, counting multiplicities, precisely $n$ complex roots. For instance, constant functions have a root of order $n$ at $\infty$.) The reader can readily check that the above definition of order is invariant under general linear fractional transformations.

Lemma 5. Consider the action (3.3) of the group GL(2) on the Lie algebra $\mathfrak{h}_{n}$. If $\mathscr{H}(w)$ is a quasi-exactly solvable operator determined, as in (2.9), by the triple of polynomials $P(w), Q(w), R$, then the transformed differential operator

$$
\hat{\mathscr{H}}(z)=(\gamma z+\delta)^{n} \cdot \mathscr{H}\left(\frac{\alpha z+\beta}{\gamma z+\delta}\right) \cdot(\gamma z+\delta)^{-n},
$$

will be determined by the triple $\hat{P}(z), \hat{Q}(z), \hat{R}$, where $\hat{R}=R$, and

$$
\hat{P}(z)=\frac{(\gamma z+\delta)^{4}}{\Delta^{2}} P\left(\frac{\alpha z+\beta}{\gamma z+\delta}\right), \quad \hat{Q}(z)=\frac{(\gamma z+\delta)^{2}}{\Delta} Q\left(\frac{\alpha z+\beta}{\gamma z+\delta}\right) .
$$

Moreover, the associated module for $\mathfrak{h}_{n}$, which is just the space of polynomials $\mathscr{P}^{(n)}$, transforms according to the representation $\rho_{n, 0}$; in particular, the eigenfunctions $\hat{\chi}_{k}$ of the transformed operator $\hat{\mathscr{H}}$ are also polynomials, given by

$$
\hat{\chi}_{k}(z)=(\gamma z+\delta)^{n} \chi_{k}\left(\frac{\alpha z+\beta}{\gamma z+\delta}\right) .
$$

If we restrict to $\mathrm{SL}(2)$ (i.e., $\Delta=1$ ) the induced representation on the space of quasi-exactly solvable second order differential operators is just the direct sum of its usual representations on quartic, quadratic and constant polynomials. The full representation of GL(2) on the space of quasi-exactly solvable second order differential operators is isomorphic, via (3.6), to the sum of three irreducible representations, $\rho_{4,-2} \oplus \rho_{2,-1} \oplus \rho_{0,0}$, where $\rho_{0,0}=\mathbb{1}$ is the identity representation, reflecting the invariance of the constant $R(z)=c^{*}$.

Finally, we should remark that there is an additional obvious scaling transformation, $(P, Q, R) \mapsto(v P, v Q, v R)$, which can be used to rescale the eigenvalues of the quasi-exactly solvable operator without affecting the eigenfunctions.

Using the action of GL(2) on the space of quasi-exactly solvable Schrödinger operators, we can place the gauged operator (2.9) into a simpler canonical form, based on the invariant theoretic classification of canonical forms for quartic polynomials, [11].

Theorem 6. Under the representation $\rho_{4,-2}$ of $\mathrm{GL}(2)$, every real quartic polynomial $P(z)$ is equivalent to one of the following canonical forms:

$$
\begin{aligned}
& \text { Ia. } v\left(z^{4}+\tau z^{2}+1\right), \quad \tau \neq \pm 2, \\
& \text { Ib. } v\left(z^{4}+\tau z^{2}-1\right) \\
& \text { IIa. } v\left(z^{2}+1\right) \\
& \text { IIb. } v\left(z^{2}-1\right)
\end{aligned}
$$




$$
\begin{aligned}
& \text { III } . v z^{2}, \\
& \text { IIIb. } v\left(z^{2}+1\right)^{2}, \\
& \text { IV. } z, \\
& \text { V. } 1, 1, \\
& \text { VI. } 0,
\end{aligned}
$$

where $v$ and $\tau$ are real constants.

In case I, all roots (real or complex) are simple, while cases II-V have one or more multiple roots. Case Ia, for $\tau<-2$ has four real roots (and the sign can be taken to be positive without loss of generality), whereas for $\tau>-2$ all four roots are complex. Case Ib has two real and two complex-conjugate roots. Case II has a double root at $\infty$ and either two simple real roots (if the signs are opposite) or two simple complex roots (if the signs are the same). Case III has two double roots; for IIIa they are at 0 and $\infty$, while for IIIb they are at $\pm i$. Cases IV and V have, respectively, a triple or a quadruple root at $\infty$. See [11; Exercises 25.13, 25.14] for more details, Cases III-V (we exclude the trivial case VI throughout, as the associated differential operator (2.9) is only first order) have a residual oneparameter symmetry group which can be used to simplify the form of the quadratic polynomial $Q(z)$, but this will not be required.

A similar classification holds for the representations $\rho_{4, i}$ with $i \neq-2$, but there is an additional scaling which allows us to reduce the parameter $v$ in the first three cases to \pm 1 . The distinguished role of the particular weighting $(4,-2)$ is quite interesting and will be remarked upon again later.

\section{Gauge Transformations and Asymptotics}

As discussed in [12], the gauge transformation (change of variables) required to place the Hamiltonian operator (2.9) into Schrödinger form can be explicitly constructed using (in general) elliptic functions.

Theorem 7. Let

$$
-\mathscr{H}=P(z) D_{z}^{2}+\left\{Q(z)-\frac{n-1}{2} P^{\prime}(z)\right\} D_{z}+\left\{R-\frac{n}{2} Q^{\prime}(z)+\frac{n(n-1)}{12} P^{\prime \prime}(z)\right\}
$$

be a quasi-exactly solvable differential operator. Assume $P(z)>0$ on an interval $I \subset \mathbb{R}$. Define the change of variables $z=\zeta(x)$ where $(d z / d x)^{2}=P(z)$, with inverse given by the elliptic integral

$$
x=\xi(z)=\int^{z} \frac{d y}{\sqrt{P(y)}}
$$

for $z \in I$. Further, define the function

$$
\mu(z)=|P(z)|^{-n / 4} \exp \left\{\int^{z} \frac{Q(y)}{2 P(y)} d y\right\}
$$


Then the change of variables $z=\zeta(x)$ and gauge factor $\mu(z)$ will place the operator $\mathscr{H}$ into Schrödinger form

$$
\mu(z) \cdot \mathscr{H} \cdot \frac{1}{\mu(z)}=-D_{x}^{2}+V(x)
$$

The potential is given by

$$
V(x)=-\frac{n(n+2)\left(P P^{\prime \prime}-\frac{3}{4} P^{\prime 2}\right)+3(n+1)\left(Q P^{\prime}-2 P Q^{\prime}\right)-3 Q^{2}}{12 P}-R,
$$

where the right-hand side is evaluated at $z=\zeta(x)$. Moreover, if $\chi(z)$ is a wave function in the original coordinates, then

$$
\psi(x)=\mu(\zeta(x)) \chi(\zeta(x))
$$

will be the corresponding wave function in the physical (Schrödinger) coordinates.

The asymptotics of the wave functions in the physical coordinates will be determined by the roots of the quartic polynomial $P(z)$, since if $z$ is finite, and $P(z)>0$, then the change of variables $x=\xi(z)$ will be a smooth, locally monotone function near $z$. Each maximal interval of positivity of $P$ will give a different interval of definition of the physical variable $x$, so a given quasi-exactly solvable operator in the $z$ coordinates can lead to more than one quasi-exactly solvable Schrödinger operator! The intervals themselves will be bounded by either a root $z_{0}$ of $P(z)$ or by $z_{0}= \pm \infty$, and the corresponding asymptotic behavior of the change of variables $z=\zeta(x)$ will be determined by the leading order behavior of $P(z)$ as $z \rightarrow z_{0}$. The following elementary lemma provides a complete description of the asymptotics.

Lemma 8. Suppose $z=\zeta(x)$ is a real-valued function satisfying the ordinary differential equation $z^{\prime 2}=P(z)$. Suppose that, as $x \rightarrow x_{0}$, we have $z=\zeta(x) \rightarrow z_{0}$, where $z_{0}$ is $a$ (finite) root of $P$. Let $\varepsilon= \pm 1$ correspond to the side we approach $z_{0}$ from, i.e., $\varepsilon=+1$ if $z \rightarrow z_{0}^{+}$, and $\varepsilon=-1$ if $z \rightarrow z_{0}^{-}$. Suppose $P$ has the asymptotic behavior

$$
P(z) \sim \alpha\left|z-z_{0}\right|^{m}, \quad \text { as } z \rightarrow z_{0},
$$

for some $\alpha>0$. (Positivity is required in order that the solution $\zeta(x)$ be real.) For $m \neq 2$, define

$$
\rho=\frac{2}{2-m}, \quad \beta=\frac{|\alpha|^{\rho / 2}}{|\rho|^{\rho}} .
$$

Then the limit point $x_{0}$ is finite if and only if $m<2$, in which case

$$
\zeta(x)-z_{0} \sim \varepsilon \beta\left|x-x_{0}\right|^{\rho}, \quad x \rightarrow x_{0}, \quad m<2 .
$$

If $m \geqq 2$, then $x_{0}= \pm \infty$, and

$$
\zeta(x)-z_{0} \sim\left\{\begin{array}{ll}
\varepsilon \beta|x+\kappa|^{\rho}, & m>2, \\
\varepsilon e^{-\sqrt{\alpha}|x+\kappa|,} & m=2,
\end{array}|x| \rightarrow \infty,\right.
$$

for some phase shift $\kappa$.

Similarly, suppose $z_{0}=\varepsilon \infty$, is infinite, with $\varepsilon= \pm 1$, and assume $z=\zeta(x) \rightarrow \varepsilon \infty$ as $x \rightarrow x_{0}$. If $P(z) \sim \alpha|z|^{m}$ as $z \rightarrow z_{0}$, then the limit point $x_{0}$ is finite if and only if $m>2$, in which case $\zeta(x) \sim \varepsilon \beta\left|x-x_{0}\right|^{\rho}$. 


\section{Normalizability of Wave Functions and Normalizable Potentials}

Recall that in the quantum mechanical interpretation, a nonzero wave function $\psi(x)$, defined on all of $\mathbb{R}$, is called normalizable if it is square integrable, since in this case it can be normalized to determine a genuine probability distribution $\psi(x) /\|\psi\|$ on $\mathbb{R}$ by dividing by its norm. For a quasi-exactly solvable Hamiltonian corresponding to the Lie algebra $\mathfrak{h}_{n}$, the eigenfunctions obtained by algebraic methods are given by polynomials $\chi_{k}(z)$ of degree at most $n$ in the $z$-coordinates. According to (4.5), the corresponding eigenfunctions in the physical $x$-coordinates are

$$
\psi_{k}(x)=\mu(z) \chi_{k}(z)=\mu(\zeta(x)) \chi_{k}(\zeta(x))
$$

where each $\chi_{k}(z)$ is a polynomial in $z$ of maximal degree $k$, and $z=\zeta(x)$ is the change of variables described by (4.2). Since they are determined using algebraic techniques, we will refer to the functions $\psi_{k}(x)$ as algebraic eigenfunctions for short; this does not mean that the functions are algebraic in the traditional, mathematical sense of the word.

The normalizability problem is to determine whether or not the algebraic eigenfunctions (5.1) are in the Hilbert space $\mathrm{L}^{2}=\mathrm{L}^{2}(\mathbb{R})$. Three obvious sources of difficulty can arise in this regards. First, the change of variables $x=\xi(z)$ may not cover the entire real $x$-space. Second, the gauge factor $\mu(\zeta(x))$, defined by (4.3), could become singular for some finite value $x_{0}$ of $x$. Third, the potential, as defined by (4.4), could itself have a singularity. Clearly these latter two possibilities will not happen unless $z_{0}=\zeta\left(x_{0}\right)$ is a root of $P$, but they can happen at simple roots of $P$, as we shall shortly see. The first difficulty is dealt with by the following elementary result.

Lemma 9. Suppose $P(z)>0$ is positive on the open interval $z_{0}<z<z_{1}$, and vanishes at the endpoints $P\left(z_{0}\right)=P\left(z_{1}\right)=0$. If both $z_{0}$ and $z_{1}$ are simple roots, then none of the physical eigenfunctions (5.1) are normalizable on $\mathbb{R}$.

Proof. If both $z_{0}$ and $z_{1}$ are simple roots, then the inverse $z=\zeta(x)$ of the elliptic integral (4.2), i.e., the solution to the ordinary differential equation

$$
\zeta^{\prime 2}=P(\zeta)
$$

will be a periodic function of $x$, going back and forth between its minimum $z_{0}$ and maximum $z_{1}$. Consequently, all eigenfunctions (5.1) will also be periodic functions of $x$, and hence can never be normalizable. (On the other hand, they will contribute to the continuous spectrum of the Schrödinger operator, and therefore, provided they are bounded, determine a (very small) part of the scattering data. We will discuss this case in a subsequent paper.) Q.E.D.

Suppose the conditions of Lemma 9 hold. If $z_{0}$ is a multiple root and $z_{1}$ a simple root, then $\zeta(x)$ will be defined on the real line, being asymptotic to $z_{0}$ as $|x| \rightarrow \infty$, and monotone, symmetric around its maximum value $z_{1}$. A similar behavior holds if $z_{1}$ is the multiple root, with $\zeta(x)$ having a minimum $z_{0}$ and asymptotic to $z_{1}$ at $\pm \infty$. Finally, if both $z_{0}$ and $z_{1}$ are multiple roots, then $\zeta(x)$ will be a monotone function on $\mathbb{R}$, asymptotic to $z_{0}$ at one end and $z_{1}$ at the other. These remarks also hold if $z_{0}$ or $z_{1}$ is $\infty$. Moreover, if $P$ has degree 4 , so $\infty$ is not a root according to our earlier definition, then we can "integrate through $\infty$ " from a finite root $z_{0}$ to 
another finite root $z_{1} \leqq z_{0}$. (Alternatively, we can perform a linear fractional transformation so that $\infty$ is no longer in the interval under consideration.) Finally, if $P(z)>0$ is positive definite, and has no roots (finite or infinite), then, even though we can continue to integrate through $\infty$, we can never produce normalizable wave functions.

Summarizing the preceding discussion, only quartics with at least one multiple root are possible candidates for producing normalizable operators; moreover, the multiple root must lie at the end of an interval of positivity of the quartic $P(z)$. Therefore, the only canonical quartics from Theorem 6 which can possibly produce normalizable wave functions are the following:

$$
\begin{array}{ll}
\text { 1. } P(z)=v\left(z^{2}+1\right), & (-\infty, \infty) \\
\text { 2. } P(z)=v\left(z^{2}-1\right), & {[1, \infty) \text { or }(-\infty,-1]} \\
\text { 3. } P(z)=v z^{2}, & (0, \infty) \text { or }(-\infty, 0) \\
\text { 4. } P(z)=z, & {[0, \infty)} \\
\text { 5. } P(z)=1 . & (-\infty, \infty)
\end{array}
$$

The second column indicates the interval(s) where $P$ is positive, with square brackets indicating simple roots. In case $1, P$ has a single double root at $\infty$; in case $2, P$ has a double root at $\infty$ and a pair of simple roots at \pm 1 ; in case $3, P$ has a double root at 0 and a double root at $\infty$; in case $4, P$ has a triple root at $\infty$ and a simple root at 0 ; in case $5, P$ has a single quadruple root at $\infty$, and no change of independent variable is required. In cases 2 and 3 there are two possible intervals of positivity, and hence potentially two different physical Hamiltonians, although we can readily switch from one to the other by a discrete reflection $z \mapsto-z$. As remarked in Theorem 6 , the positive multiplier $v>0$ must be included in cases 1,2 , and 3 , because we are dealing with the particular representation $\rho_{4,-2}$, although we could omit it by rescaling the operator (and hence also $Q$ and $R$ ).

In cases 2 and 4 , the polynomial has a simple root at \pm 1 or 0 , which, as remarked above, introduces further complications into our normalizability problem. Choose the integration constant so that the solution $\zeta(x)$ to $(5.2)$ is symmetric about $x=0$. The restrictions of $\zeta(x)$ to the intervals $(-\infty, 0)$ or $(0, \infty)$ thus define two one-to-one mappings onto $(1, \infty),(-\infty,-1),(0, \infty)$, for cases $2 \mathrm{a}, 2 \mathrm{~b}$, and 4 , respectively. We can therefore map the canonical operator $\mathscr{H}$ into two Schrödinger operators $\mathscr{S}_{-}(x)=-D_{x}^{2}+V_{-}(x)$ and $\mathscr{S}_{+}(x)=-D_{x}^{2}+V_{+}(x)$, defined respectively on $(-\infty, 0)$ and on $(0, \infty)$. Their respective potentials satisfy

$$
V_{-}(-x)=V_{+}(x), \quad x>0,
$$

and therefore define an even function $V(x)$ on the entire real line. This potential may or may not have a singularity at the origin $x=0$. The singular case will be discussed in a separate section, so we assume now that $V(0)$ is finite. The problem is how to extend the eigenfunctions of, say, $\mathscr{S}_{+}$(obtained by diagonalizing $\mathscr{H}$ ), which are only defined for $x>0$, to eigenfunctions of $\mathscr{S}=-D_{x}^{2}+V(x)$, defined for all $x \in \mathbb{R}$. Now, since $V(x)$ is an even function, the $L^{2}$ eigenfunctions of $\mathscr{S}$ must have a well-defined parity. If $\psi_{+}(x)$ is an eigenfunction of $\mathscr{S}_{+}$defined on $(0, \infty)$, and $\lim _{x \rightarrow 0+} \psi_{+}(x)=\psi_{+}(0)$ exists, then elementary uniqueness theorems for second order ordinary differential equations imply that we can extend $\psi_{+}(x)$ to be 
a smooth, even eigenfunction, $\psi(x)=\psi(-x)$, of $\mathscr{S}$ if $\psi+(0) \neq 0$, or to be a smooth, odd eigenfunction, $\psi(x)=-\psi(-x)$, of $\mathscr{S}$ if $\psi_{+}(0)=0$.

Definition 10. A quasi-exactly solvable potential $V(x)$, defined for $x \in \mathbb{R}$, is called normalizable if every algebraic eigenfunction is normalizable. The potential is called partially normalizable if some, but not necessarily all, of the algebraic eigenfunctions are normalizable.

Tedious but direct calculations based on Theorem 7 produce the explicit change of variables, the potential, and eigenfunctions for the above normal forms in physical coordinates. Each of the five classes of potentials is a linear combination of four elementary functions, but the coefficients must satisfy a certain algebraic constraint. Analysis of the explicit formulas for the eigenfunctions yields a complete set of conditions for the normalizability and partial normalizability of the potentials.

According to the definition of [22], a potential is exactly solvable if it does not explicitly depend on the "spin" $n$, since, in this case, one can find representation spaces of arbitrarily large dimension and thereby produce infinitely many eigenvalues by algebraic methods (although there is no guarantee that these will correspond to normalizable eigenfunctions). Note that since the gauge transformation (4.2), (4.3), can explicitly depend on the parameter $n$, exact solvability cannot be detected in the canonical coordinates, but depends on the final physical, i.e., Schrödinger, form of the operator. (For instance, if we included a fixed, nonzero magnetic field, corresponding to a first order differential operator term, in our earlier definition of a Schrödinger operator, we would have a different set of conditions for exact solvability.) We also exhibit a complete list of one-dimensional exactly solvable, normalizable potentials, which includes the well known harmonic oscillator, radial harmonic oscillator, Morse, and (restricted) Pöschl-Teller potentials, as well as a number of new and interesting cases not noted before in the literature

Canonical forms for Quasi-exactly Solvable Spectral Problems

Case 1. $P(z)=v\left(z^{2}+1\right), z \in(-\infty, \infty)$.

Change of variables: $z=\sinh \sqrt{v} x$.

Gauge factor:

$$
\begin{aligned}
\mu(z) & =\left(z^{2}+1\right)^{-\frac{n}{4}} \exp \left\{\frac{1}{2 v} \int \frac{\hat{b} z^{2}+\hat{c} z+\hat{d}}{z^{2}+1} d z\right\} \\
& =\left(z^{2}+1\right)^{\frac{\hat{c}}{4 v}-\frac{n}{4}} \exp \left[\frac{\hat{b} z+(\hat{d}-\hat{b}) \tan ^{-1} z}{2 v}\right] .
\end{aligned}
$$

Potential:

$$
V(x)=A \sinh ^{2} \sqrt{v} x+B \sinh \sqrt{v} x+C \tanh \sqrt{v} x \operatorname{sech} \sqrt{v} x+D \operatorname{sech}^{2} \sqrt{v} x,
$$

where

$$
\begin{aligned}
A=\frac{\hat{b}^{2}}{4 v}, \quad B & =\frac{(\hat{c}+(n+1) v) \hat{b}}{2 v}, \quad C=\frac{(\hat{c}-(n+1) v)(\hat{d}-\hat{b})}{2 v}, \\
D & =\frac{(\hat{d}-\hat{b})^{2}-(\hat{c}-(n+1) v)^{2}+v^{2}}{4 v} .
\end{aligned}
$$


Constraints:

$$
[B \pm 2(n+1) \sqrt{v A}]^{4}+A(4 D-v)[B \pm 2(n+1) \sqrt{v A}]^{2}-4 A^{2} C^{2}=0, \quad A \geqq 0 .
$$

Exactly Solvable Potentials:

$$
V(x)=C \tanh \sqrt{v} x \operatorname{sech} \sqrt{v} x+D \operatorname{sech}^{2} \sqrt{v} x .
$$

These include the restricted Pöschl-Teller potentials

$$
V(x)=D \operatorname{sech}^{2} \sqrt{v} x, \quad D<0 .
$$

In particular, if $D=-m(m+1)$, where $m$ is a non-negative integer, than $V(x)$ is a reflectionless $m$-soliton potential.

Eigenfunctions:

$$
\psi_{k}(x)=(\cosh \sqrt{v} x)^{\frac{\hat{c}}{2 v}-\frac{n}{2}} \exp \left[\frac{\hat{b} \sinh \sqrt{v} x+(\hat{d}-\hat{b}) \operatorname{gd} \sqrt{v} x}{2 v}\right] \chi_{k}(\sinh \sqrt{v} x)
$$

where $\operatorname{gd} x=2 \tan ^{-1} e^{x}-\frac{\pi}{2}=\tan ^{-1} \sinh x$ is the Gudermannian or hyperbolic amplitude function, [10; p. 43].

Partial normalizability conditions:

$$
\hat{b}=0, \quad \text { and } \quad \operatorname{deg} \chi_{k}<\frac{n}{2}-\frac{\hat{c}}{2 v} .
$$

Full normalizability conditions:

$$
\hat{b}=0, \text { and } \hat{c}<-n v .
$$

Note particularly that the normalizability conditions will, in most cases, serve to simplify the constraint conditions, (5.6), on the coefficients for a potential to be quasi-exactly solvable. Note that in this case all normalizable potentials are exactly solvable.

For example, consider the Pöschl-Teller potential (5.8). Assume that we have partial normalizability conditions (5.9) holding for one or more algebraic eigenfunctions. In this case, according to $(5.5), 0=2 v C=\hat{d}(\hat{c}-v(n+1))$. However, the second normalizability condition in (5.9) implies that $\hat{c}-v(n+1) \neq 0$, and therefore we must have $\hat{d}=0$. The potential is then of the form $V=-\frac{1}{4} v K \operatorname{sech}^{2}$ $\sqrt{v} x$ with

$$
K=-4 \frac{D}{v}=\left(\frac{\hat{c}}{v}-(n+1)\right)^{2}-1>0,
$$

the inequality following from (5.9). Solving for $\hat{c}$ in terms of the potential strength $K$ we obtain

$$
\frac{\hat{c}}{v}=n+1-\sqrt{K+1}
$$


where we have taken (5.9) into account to determine the appropriate sign in front of the square root. The normalizability condition (5.9) can therefore be expressed in terms of the potential strength $K$ as follows

$$
\operatorname{deg} \chi_{k}<\frac{1}{2}(\sqrt{K+1}-1)
$$

The number of algebraic eigenstates is equal to one plus the highest value of the degree of $\chi_{k}$, which is therefore given by the integer

$$
\left[\frac{1+\sqrt{K+1}}{2}\right]
$$

where $[x]=\max \{m \in \mathbb{N} \mid m<x\}$. This reproduces the well-known number of bound states of the potential (5.8), [13; p. 74], and shows (for the first time, we believe) that all the eigenvalues of the Pöschl-Teller potential can be computed algebraically.

Case 2a. $P(z)=v\left(z^{2}-1\right), z \in[1, \infty)$.

Change of variables: $z=\cosh \sqrt{v} x$.

Gauge factor:

$$
\begin{aligned}
\mu(z) & =\left(z^{2}-1\right)^{-\frac{n}{4}} \exp \left\{\frac{1}{2 v} \int \frac{\hat{b} z^{2}+\hat{c} z+\hat{d}}{z^{2}-1} d z\right\} \\
& =\left(z^{2}-1\right)^{\frac{\hat{c}}{4 v}-\frac{n}{4}} \exp \left[\frac{\hat{b} z-(\hat{d}+\hat{b}) \operatorname{coth}^{-1} z}{2 v}\right] .
\end{aligned}
$$

Potential:

$$
V(x)=A \cosh ^{2} \sqrt{v} x+B \cosh \sqrt{v} x+C \operatorname{coth} \sqrt{v} x \operatorname{csch} \sqrt{v} x+D \operatorname{csch}^{2} \sqrt{v} x,
$$

where

$$
\begin{gathered}
A=\frac{\hat{b}^{2}}{4 v}, \quad B=\frac{(\hat{c}+(n+1) v) \hat{b}}{2 v}, \quad C=\frac{(\hat{c}-(n+1) v)(\hat{b}+\hat{d})}{2 v}, \\
D=\frac{(\hat{b}+\hat{d})^{2}+(\hat{c}-(n+1) v)^{2}-v^{2}}{4 v} .
\end{gathered}
$$

The potential has a singularity at $x=0$ unless $C+D=0$. Since

$$
C+D=\frac{(\hat{b}+\hat{c}+\hat{d}-n v)(\hat{b}+\hat{c}+\hat{d}-(n+2) v)}{4 v},
$$

the potential is regular at the origin if and only if

$$
\hat{b}+\hat{c}+\hat{d}=n v \quad \text { or } \quad \hat{b}+\hat{c}+\hat{d}=(n+2) v .
$$

Constraints:

$[B \pm 2(n+1) \sqrt{v A}]^{4}-A(4 D+v)[B \pm 2(n+1) \sqrt{v A}]^{2}+4 A^{2} C^{2}=0, \quad A \geqq 0$. 
Exactly Solvable Potentials:

$$
V(x)=C \operatorname{coth} \sqrt{v} x \operatorname{csch} \sqrt{v} x+D \operatorname{csch}^{2} \sqrt{v} x, \quad|C| \leqq D+\frac{1}{4} v .
$$

The nonsingular cases, i.e., when $C+D=0, D>0$ provide, we believe, significant new examples of exactly solvable potentials.

The algebraic eigenfunctions for the general, possibly singular, quasi-exactly solvable potential (5.11) on the half line $x>0$ take the form

$$
\psi_{k,+}(x)=(\sinh \sqrt{v} x)^{\frac{\hat{c}}{2 v}-\frac{n}{2}}\left(\tanh \sqrt{v} \frac{x}{2}\right)^{\frac{\hat{b}+\hat{d}}{2 v}} \exp \left[\frac{\hat{b}}{2 v} \cosh \sqrt{v} x\right] \chi_{k}(\cosh \sqrt{v} x) .
$$

Note that $\psi_{k,+}(x)$ is singular at $x=0$ if $4 v \mathcal{O}\left(\chi_{k}, 1\right)<n v-\hat{b}-\hat{c}-\hat{d}$.

For the two nonsingular cases, (5.12), the algebraic eigenfunctions are all nonsingular at $x=0$, and so can be extended to the entire real line. If $\hat{b}+\hat{c}+\hat{d}$ $=n v$, then we find even eigenfunctions

$$
\psi_{k}(x)=\left(\cosh \sqrt{v} \frac{x}{2}\right)^{\frac{\hat{c}}{v}-n} \exp \left(\frac{\hat{b}}{2 v} \cosh \sqrt{v} x\right) \chi_{k}(\cosh \sqrt{v} x), \quad x \in \mathbb{R} .
$$

on the entire line; on the other hand, when $\hat{b}+\hat{c}+\hat{d}=(n+2) v$, we find odd eigenfunctions

$\psi_{k}(x)=\sinh \sqrt{v} \frac{x}{2}\left(\cosh \sqrt{v} \frac{x}{2}\right)^{\frac{\hat{c}}{v}-n-1} \exp \left(\frac{\hat{b}}{2 v} \cosh \sqrt{v} x\right) \chi_{k}(\cosh \sqrt{v} x), \quad x \in \mathbb{R}$.

Partial normalizability conditions: The regularity conditions (5.12) and either

$$
\hat{b}<0 \text {, }
$$

or

$$
\hat{b}=0, \quad \text { and } \quad \operatorname{deg} \chi_{k}<\frac{n}{2}-\frac{\hat{c}}{2 v} .
$$

Full normalizability conditions: The regularity conditions (5.12) and either (5.14) or

$$
\hat{b}=0, \text { and } \hat{c}<-n v \text {. }
$$

Case 2b. $P(z)=v\left(z^{2}-1\right), z \in(-\infty,-1]$.

This case is just like Case $2 \mathrm{a}$, with $z$ replaced by $-z$. Thus the change of variables is $z=-\cosh \sqrt{v} x$, and the formulas for the gauge factor, potential, etc. are found from those in Case $2 \mathrm{a}$ by replacing $z$ by $-z$ and/or cosh by $-\cosh$. In particular, the potential is the same as (5.11) with $B, C$ replaced by $-B,-C$, and is regular at the origin if and only if

$$
-\hat{b}+\hat{c}-\hat{d}=n v \text { or } \quad-\hat{b}+\hat{c}-\hat{d}=(n+2) v .
$$

Partial normalizability conditions: The regularity conditions (5.17) and either (5.15) or

$$
\hat{b}>0 \text {. }
$$


Full normalizability conditions: The regularity conditions (5.17) and either (5.18) or (5.16).

Case 3a. $P(z)=v z^{2}, z \in(0, \infty)$.

Change of variables: $z=e^{\sqrt{v} x}$.

Gauge factor:

$$
\mu(z)=z^{-\frac{n}{2}} \exp \left\{\frac{1}{2 v} \int \frac{\hat{b} z^{2}+\hat{c} z+\hat{d}}{z^{2}} d z\right\}=z^{\frac{\hat{c}}{2 v}-\frac{n}{2}} \exp \left[\frac{\hat{b} z}{2 v}-\frac{\hat{d}}{2 v z}\right] .
$$

Potential:

$$
V(x)=A e^{2 \sqrt{v} x}+B e^{\sqrt{v} x}+C e^{-\sqrt{v} x}+D e^{-2 \sqrt{v} x},
$$

where

$$
A=\frac{\hat{b}^{2}}{4 v}, \quad B=\frac{(\hat{c}+(n+1) v) \hat{b}}{2 v}, \quad C=\frac{(\hat{c}-(n+1) v) \hat{d}}{2 v}, \quad D=\frac{\hat{d}^{2}}{4 v} .
$$

Constraints:

$$
\pm C \sqrt{A} \pm B \sqrt{D}=2(n+1) \sqrt{v A D}, \quad A, D \geqq 0 .
$$

Exactly Solvable Potentials:

$$
V(x)=A e^{2 \sqrt{v} x}+B e^{\sqrt{v} x}, \text { or } \quad V(x)=C e^{-\sqrt{v} x}+D e^{-2 \sqrt{v} x} .
$$

These are the Morse potentials.

Eigenfunctions:

$$
\psi_{k}(x)=\exp \left[\frac{\hat{b}}{2 v} e^{\sqrt{v} x}+\frac{\hat{c}-n v}{2 \sqrt{v}} x-\frac{\hat{d}}{2 v} e^{-\sqrt{v} x}\right] \chi_{k}\left(e^{\sqrt{v} x}\right) .
$$

Partial normalizability conditions:

$$
\hat{b}<0, \quad \text { or } \quad \hat{b}=0, \quad \operatorname{deg} \chi_{k}<\frac{n}{2}-\frac{\hat{c}}{2 v},
$$

and

$$
\hat{d}>0, \quad \text { or } \quad \hat{d}=0, \quad \mathcal{O}\left(\chi_{k}, 0\right)>\frac{n}{2}-\frac{\hat{c}}{2 v} .
$$

Full normalizability conditions:

$$
\hat{b}<0, \quad \text { or } \hat{b}=0, \quad \hat{c}<-n v,
$$

and

$$
\hat{d}>0, \quad \text { or } \hat{d}=0, \quad \hat{c}>n v .
$$

Note that we cannot have both $\hat{b}=0$ and $\hat{d}=0$.

For example, consider a Morse potential (5.21) with (for instance) $C=-2 D$. According to $(5.5), \hat{b}=0, \hat{d}=v(n+1)-\hat{c}$, so the potential is

$$
V(x)=v K\left(e^{-2 \sqrt{v} x}-2 e^{-\sqrt{v} x}\right),
$$


with $\hat{d}$ and $K>0$ related by $\hat{d}=2 v \sqrt{K}$, which relies on the first normalizability condition in (5.23). The second normalizability condition in (5.22) then easily reduces to

$$
\operatorname{deg} \chi_{k}<\sqrt{K}-\frac{1}{2}
$$

Hence in this case the maximum number of algebraically computable eigenfunctions is given by the integer $\left[\sqrt{K}+\frac{1}{2}\right]$, which again exactly coincides with the known number of bound states for the potential $(5.26),[13 ;$ p. 73$]$.

Case $3 b . P(z)=v z^{2}, z \in(-\infty, 0)$.

Change of variables: $z=-e^{\sqrt{v} x}$.

Gauge factor:

$$
\mu(z)=|z|^{-\frac{n}{2}} \exp \left\{\frac{1}{2 v} \int \frac{\hat{b} z^{2}+\hat{c} z+\hat{d}}{z^{2}} d z\right\}=|z|^{\frac{\hat{c}}{2 v}-\frac{n}{2}} \exp \left[\frac{\hat{b} z}{2 v}-\frac{\hat{d}}{2 v z}\right] .
$$

The potential is the same as that in Case $3 \mathrm{a}$ with $B, C$ replaced by $-B,-C$.

Eigenfunctions:

$$
\psi_{k}(x)=\exp \left[-\frac{\hat{b}}{2 v} e^{\sqrt{v} x}+\frac{\hat{c}-n v}{2 \sqrt{v}} x+\frac{\hat{d}}{2 v} e^{-\sqrt{v} x}\right] \chi_{k}\left(-e^{\sqrt{v} x}\right) .
$$

Partial normalizability conditions:

$$
\hat{b}>0, \quad \text { or } \quad \hat{b}=0, \quad \operatorname{deg} \chi_{k}<\frac{n}{2}-\frac{\hat{c}}{2 v},
$$

and

$$
\hat{d}<0, \quad \text { or } \quad \hat{d}=0, \quad \mathcal{O}\left(\chi_{k}, 0\right)>\frac{n}{2}-\frac{\hat{c}}{2 v} .
$$

Full normalizability conditions:

$$
\hat{b}<0, \quad \text { or } \hat{b}=0, \quad \hat{c}<-n v,
$$

and

$$
\hat{d}>0, \quad \text { or } \quad \hat{d}=0, \quad \hat{c}>n v .
$$

Case 4. $P(z)=z, z \in[0, \infty)$.

Change of variables: $z=\frac{1}{4} x^{2}$.

Gauge factor:

$$
\mu(z)=z^{-\frac{n}{4}} \exp \left\{\frac{1}{2} \int \frac{\hat{b} z^{2}+\hat{c} z+\hat{d}}{z} d z\right\}=z^{\frac{2 \hat{d}-n}{4}} \exp \left[\frac{1}{4} \hat{b} z^{2}+\frac{1}{2} \hat{c} z\right] .
$$

Potential:

$$
V(x)=A x^{6}+B x^{4}+C x^{2}+\frac{D}{x^{2}},
$$


where

$$
\begin{gathered}
A=\frac{1}{256} \hat{b}^{2}, \quad B=\frac{1}{32} \hat{b} \hat{c}, \quad C=\frac{1}{16}\left[\hat{c}^{2}+(2 \hat{d}+3(n+1)) \hat{b}\right], \\
D=\left(\hat{d}-\frac{n}{2}\right)\left(\hat{d}-\frac{n}{2}-1\right) .
\end{gathered}
$$

Constraints:

$$
\begin{gathered}
16 A^{3}[(4 n+5)(4 n+3)-4 D] \pm 32(n+1) A^{3 / 2}\left(B^{2}-4 A C\right)+\left(B^{2}-4 A C\right)^{2}=0, \\
A \geqq 0, \quad D \geqq-\frac{1}{4} .
\end{gathered}
$$

Note that $V$ is singular at the origin unless $D=0$, so the conditions

$$
\hat{d}=\frac{n}{2} \quad \text { or } \quad \hat{d}=\frac{n}{2}+1
$$

are necessary and sufficient for regularity. The resulting potentials are the anharmonic oscillator potentials discussed in detail in $[22,20]$.

Exactly Solvable Potentials:

$$
V(x)=C x^{2}+\frac{D}{x^{2}}, \quad C \geqq 0, \quad D \geqq-\frac{1}{4} .
$$

These are radial harmonic oscillator potentials when $D=l(l+1), l \in \mathbb{N}$.

Eigenfunctions: The algebraic eigenfunctions for the general, possibly singular, quasi-exactly solvable potential (5.31) on the half line $x>0$ take the form

$$
\psi_{k,+}(x)=x^{\hat{d}-\frac{n}{2}} \exp \left[\frac{\hat{b}}{64} x^{4}+\frac{\hat{c}}{8} x^{2}\right] \chi_{k}\left(\frac{x^{2}}{4}\right) .
$$

In the two nonsingular cases, (5.32), if $\hat{d}=\frac{n}{2}$, then the algebraic eigenfunctions can be extended to give even eigenfunctions

$$
\psi_{k}(x)=\exp \left(\frac{\hat{b}}{64} x^{4}+\frac{\hat{c}}{8} x^{2}\right) \chi_{k}\left(\frac{x^{2}}{4}\right), \quad x \in \mathbb{R}
$$

on the entire line. On the other hand, when $\hat{d}=\frac{n}{2}+1$ the algebraic eigenfunctions can be extended to give odd eigenfunctions

$$
\psi_{k}(x)=x \exp \left(\frac{\hat{b}}{64} x^{4}+\frac{\hat{c}}{8} x^{2}\right) \chi_{k}\left(\frac{x^{2}}{4}\right), \quad x \in \mathbb{R} .
$$

Normalizability conditions: (5.32) and

$$
\hat{b}<0, \quad \text { or } \hat{b}=0, \quad \hat{c}<0 \text {. }
$$


In this case, there are no partially normalizable, regular potentials.

Case 5. $P(z)=1, z \in(-\infty, \infty)$.

Change of variables: $z=x$.

Gauge factor:

$$
\mu(z)=\exp \left[\frac{1}{6} \hat{b} z^{3}+\frac{1}{4} \hat{c} z^{2}+\frac{1}{2} \hat{d} z\right] .
$$

Potential:

$$
V(x)=A x^{4}+B x^{3}+C x^{2}+D x
$$

where

$$
A=\frac{1}{4} \hat{b}^{2}, \quad B=\frac{1}{2} \hat{b} \hat{c}, \quad C=\frac{1}{4}\left[\hat{c}^{2}+2 \hat{b} \hat{d}\right], \quad D=\frac{1}{2}[2 \hat{b}(n+1)+\hat{c} \hat{d}] .
$$

Constraints:

$$
8 A^{2} D+B\left(B^{2}-4 A C\right)= \pm 16(n+1) A^{5 / 2}, \quad A \geqq 0 .
$$

Exactly Solvable Potentials:

$$
V(x)=C x^{2}+D x, \quad C \geqq 0 .
$$

These are (translated) harmonic oscillator potentials.

Eigenfunctions:

$$
\psi_{k}(x)=\exp \left[\frac{1}{6} \hat{b} x^{3}+\frac{1}{4} \hat{c} x^{2}+\frac{1}{2} \hat{d} x\right] \chi_{k}(x)
$$

Normalizability conditions:

$$
\hat{b}=0, \text { and } \hat{c}<0 .
$$

Note that, in this case, there are no partially normalizable cases; moreover, every normalizable quasi-exactly solvable potential is just a translate of the harmonic oscillator, and is hence exactly solvable.

\section{Invariant Characterization of Normalizability}

The results of the preceding section give a complete solution to our normalizability problem. The only draw-back is that, in order to apply them, one first needs to place the quasi-exactly solvable operator in canonical form via a suitably chosen change of variables, and this requires explicit knowledge of the roots of the quartic polynomial $P$. Although, in principle, this can be done using Ferrari's formula, in practice it is rather difficult to see which particular choices of coefficients $c_{a b}$ in the original form (2.3) will indeed give rise to normalizable operators. However, since the normalizability conditions do not, obviously, depend on the choice of gauged coordinate $z$, they should be invariant under the induced action of the group GL(2) discussed in Sect. 3. Therefore, the normalizability conditions should be directly expressible in terms of invariant quantities, namely the classical joint invariants 
and covariants of the system of polynomials (2.7). The consequent invariant normalizability conditions will then provide explicit normalizability conditions on the coefficients $c_{a b}$, and the reduction to canonical form becomes unnecessary. For example, in all cases for normalizable wave functions, the quartic polynomial $P$ must have a multiple root, and this can be expressed in an invariant manner by the vanishing of the discriminant of $P$, which results in an algebraic constraint (of degree 6) in the coefficients $c_{a b}$.

In order to construct a complete system of invariant and covariants of a (system of) polynomials, the invariant-theoretic process of "transvection" is essential. We begin by defining the classical transvectants between functions (polynomials), conveniently expressed in the projection coordinate $z$, rather than the more usual homogeneous coordinates found in the classical texts.

Definition 11. Let $F(z) \in \mathscr{P}^{(m)}$ and $G(z) \in \mathscr{P}^{(n)}$ be polynomials of respective degrees (at most) $m, n$. Let $r \leqq \min \{m, n\}$. The $r^{\text {th }}$ transvectant of $F$ and $G$ is the polynomial

$$
(F, G)^{(r)}=\sum_{k=0}^{r}(-1)^{k}\left(\begin{array}{l}
r \\
k
\end{array}\right) \frac{(m-r+k) !(n-k) !}{(m-r) !(n-r) !} F^{(r-k)}(z) G^{(k)}(z) .
$$

Theorem 12. Suppose $F$ transforms under GL(2) according to the representation $\rho_{m, i}$ and $G$ transforms under GL(2) according to the representation $\rho_{n, j}$. Then the $r^{\text {th }}$ transvectant $(F, G)^{(r)} \in \mathscr{P}^{(m+n-2 r)}$ is a polynomial of degree at most $m+n-2 r$ and transforms under GL(2) according to the representation $\rho_{m+n-2 r, i+j+r}$.

The fact that $(F, G)^{(r)}$ has degree at most $m+n-2 r$ is not obvious from the formula (6.1). The proof of Theorem 12 can be deduced from the references [9] and [11]; a more direct proof will appear in a forthcoming book by the third author.

Example 13. Let $F$ have weight $(m, i)$ and $G$ weight $(n, j)$. The simplest cases of $(6.1)$ are

$$
\begin{aligned}
(F, G)^{(1)}= & n F^{\prime} G-m F G^{\prime} \\
(F, G)^{(2)}= & n(n-1) F^{\prime \prime} G-2(m-1)(n-1) F^{\prime} G^{\prime}+m(m-1) F G^{\prime \prime} \\
(F, G)^{(3)}= & n(n-1)(n-2) F^{\prime \prime \prime} G-3(m-2)(n-1)(n-2) F^{\prime \prime} G^{\prime} \\
& +3(m-1)(m-2)(n-2) F^{\prime} G^{\prime \prime}-m(m-1)(m-2) F G^{\prime \prime \prime},
\end{aligned}
$$

having weights $(m+n-2, i+j+1),(m+n-4, i+j+2),(m+n-6, i+j+3)$, respectively. In particular, the first two non-vanishing transvectants of a polynomial $F$ of degree $m$ with itself are the Hessian

$$
H[F]=\frac{1}{2 m(m-1)}(F, F)^{(2)}=F F^{\prime \prime}-\frac{m-1}{m} F^{\prime 2},
$$

of weight $(2 m-4,2 i+2)$, and the fourth transvectant

$$
\begin{aligned}
I[F] & =\frac{1}{2 m(m-1)(m-2)(m-3)}(F, F)^{(4)} \\
& =F F^{\prime \prime \prime \prime}-4 \frac{m-3}{m} F^{\prime} F^{\prime \prime \prime}+3 \frac{(m-2)(m-3)}{m(m-1)} F^{\prime \prime 2},
\end{aligned}
$$


of weight $(2 m-8,2 i+4)$. (Note that all the odd transvectants $(F, F)^{(2 s+1)}=0$ automatically vanish.)

We can use the process of transvection to construct invariants and covariants from a given polynomial. Indeed, the First Fundamental Theorem of Invariant Theory, [9], [11], says that every polynomial invariant and covariant of a system of polynomials can be constructed by successive transvection.

Example 14. Consider first the case of a single quadratic polynomial, $P(z)=a z^{2}+b z+c$. Its discriminant can be written as half the second transvectant of $P$ with itself, $\frac{1}{2}(P, P)^{(2)}=4 a c-b^{2}$, and is the only nonzero transvectant. Indeed, every covariant of a quadratic polynomial can be written in terms of the polynomial itself and its discriminant.

Example 15. For a single quartic polynomial

$$
P(z)=a z^{4}+b z^{3}+c z^{2}+d z+e,
$$

transforming (as in our case) according to the representation $\rho_{4,-2}$, a complete list of polynomially independent covariants is provided by the Hessian

$$
H=\frac{1}{24}(P, P)^{(2)}=P P^{\prime \prime}-\frac{3}{4} P^{\prime 2},
$$

which has weight $(4,-2)$, the two invariants (both of weight $(0,0))$,

$$
\begin{aligned}
i & =\frac{1}{96}(P, P)^{(4)}=\frac{1}{2} P P^{\prime \prime \prime \prime}-\frac{1}{2} P^{\prime} P^{\prime \prime \prime}+\frac{1}{4} P^{\prime \prime 2} \\
& =12 a e-3 b d+c^{2}, \\
j & =\frac{1}{96}(P, H)^{(4)}=\frac{1}{4}\left[P^{\prime \prime \prime \prime} H-P^{\prime \prime \prime} H^{\prime}+P^{\prime \prime} H^{\prime \prime}-P^{\prime} H^{\prime \prime \prime}+P H^{\prime \prime \prime \prime}\right] \\
& =\frac{1}{8} \operatorname{det}\left|\begin{array}{rrr}
12 a & 3 b & 2 c \\
3 b & 2 c & 3 d \\
2 c & 3 d & 12 e
\end{array}\right|,
\end{aligned}
$$

and the additional covariant

$$
T=\frac{1}{24}(P, H)^{(1)}=\frac{1}{6}\left[P^{\prime} H-P H^{\prime}\right]
$$

which has weight $(6,-3)$. The constants are chosen for convenience; they do not agree with most of the standard normalizations of these covariants, but, as every reference has different normalizations (even, in some cases, more than one in the same book) we feel free to choose a more convenient version which will eliminate most of the large numerical factors. We note that the discriminant of $P$ is defined to be the invariant

$$
\delta=i^{3}-j^{2}
$$


and has the basic property that $P$ has a multiple root (and so has canonical form II-VI in Theorem 6) if and only if $\delta=0$. According to the discussion in Sect. 5, then, the condition $\delta=0$, which is a sixth degree of polynomial equation

$$
\begin{aligned}
& {\left[12 c_{++} c_{--}-12 c_{+0} c_{0-}+\left(2 c_{+-}+c_{00}\right)^{2}\right]^{3}=} \\
& =\frac{1}{64}\left(\operatorname{det}\left(\begin{array}{ccc}
12 c_{++} & 6 c_{+0} & 4 c_{+-}+2 c_{00} \\
6 c_{+0} & 4 c_{+-}+2 c_{00} & 6 c_{0-} \\
4 c_{+-}+2 c_{00} & 6 c_{0-} & 12 c_{--}
\end{array}\right)^{2}\right.
\end{aligned}
$$

in the coefficients $c_{a b}$ of a quasi-exactly solvable operator (2.3), is necessary for the existence of normalizable algebraic wave functions.

Now, in the classical treatment of the invariant theory of quartic polynomials, the polynomial $P$ transforms under GL(2) according to the representation $\rho_{4,0}$, so there is no extra determinantal factor in the transformation rule. In this case the invariants $i$ and $j$ transform, respectively, according to the representations $\rho_{0,4}$ and $\rho_{0,6}$, the 0 referring to the fact that they are invariants (not covariants), and the 4 and 6 giving their respective weights. Linear combinations of invariants (and covariants) will provide further invariants, provided the summands transform according to the same representation of GL(2). Thus, the discriminant $\delta$, which has weight 12 , transforms according to the representation $\rho_{0,12}$, and is an invariant, whereas, for the classical weighting, the quantity $i-j$ is not an invariant, since the two summands transform according to different representations.

In our case, however, the situation is markedly different. The polynomial $P$ transforms under GL(2) according to the representation $\rho_{4,-2}$, cf. (3.6). Therefore, by Theorem 12, the covariants $H$ and $T$ belong to the representations $\rho_{4,-2}$ and $\rho_{6,-3}$, respectively, while the invariants $i$ and $j$ both transform according to the representations $\rho_{0,0}$, which implies that they are absolute invariants! (So is the discriminant $\delta$, and any other invariant obtained by transvection.) Now the quantity $i-j$ is an invariant, so that, for example, the condition $i=j$ has invariant meaning (albeit not very useful) for a quartic $P$ belonging to the representation space of $\rho_{4,-2}$.

The complete list of independent covariants for the system consisting of a quartic polynomial and a quadratic polynomial is known, and can be found in [9; p. 168].

Theorem 16. Let $P$ be a quartic polynomial and $Q$ a quadratic polynomial. Then a complete system of irreducible covariants for the pair $P, Q$ is provided by the polynomials themselves, the discriminant $\Delta$ of the quadratic, the covariants $H, T$, and invariants $i, j$ of the quartic, and the following 11 joint covariants:

$$
\begin{gathered}
(P, Q)^{(1)}, \quad(P, Q)^{(2)}, \quad\left(P, Q^{2}\right)^{(3)}, \quad\left(P, Q^{2}\right)^{(4)}, \quad(H, Q)^{(1)}, \quad(H, Q)^{(2)}, \\
\left(H, Q^{2}\right)^{(3)}, \quad\left(H, Q^{2}\right)^{(4)}, \quad(T, Q)^{(2)}, \quad\left(T, Q^{2}\right)^{(4)}, \quad\left(T, Q^{3}\right)^{(6)} .
\end{gathered}
$$

Of the preceding covariants, $\Delta, i, j,\left(P, Q^{2}\right)^{(4)},\left(H, Q^{2}\right)^{(4)}$, and $\left(T, Q^{3}\right)^{(6)}$ are absolute invariants, having weights $(0,0)$. Moreover, $Q,(P, Q)^{(2)},\left(P, Q^{2}\right)^{(3)}$, $(H, Q)^{(2)},\left(H, Q^{2}\right)^{(3)}$, and $\left(T, Q^{2}\right)^{(4)}$ are quadratic covariants, all of weight $(2,-1)$, whereas $P, H,(P, Q)^{(1)},(H, Q)^{(1)}$, and $(T, Q)^{(2)}$ are quartic covariants, of weight $(4,-2)$, and, finally, $T$ is a sextic covariant, of weight $(6,-3)$. Therefore we have the striking result that, when the original quartic and quadratic have weights $(4,-2)$ and $(2,-1)$, all covariants have essentially the same weightings - integral multiples of the "fundamental" weight $(2,-1)$. In particular, for this weighting, all the classical invariants are absolute invariants. (For comparison, the reader can 
compute the weights of the covariants in the "classical" weighting where $P$ has weight $(4,0)$ and $Q$ has weight $(2,0)$; they are much more irregular, leading one to believe that our weighting is, in a very definite sense, the most natural!)

Remark. Note that the potential $V(x)$ associated with a quasi-exactly solvable operator (4.1) is an absolute covariant, and hence, not surprisingly, the explicit formula (4.4) can be written in terms of the basic covariants, namely

$$
V(x)=-\frac{2 n(n+2) H(P)+3(n+1)(P, Q)^{(1)}-6 Q^{2}}{24 P}-R,
$$

where $H(P)$ is the Hessian of the quartic $P$, given by (6.5).

We are now ready to determine the invariant conditions for full normalizability. Note that, due to syzygies and redundancies among the invariants and covariants, every one of our invariant conditions has many different reformulations. We have merely chosen what appears to be the most convenient or straightforward of the various options. The computation of transvectants is readily done using a simple Mathematica program. If $P(z)$ and $Q(z)$ are polynomials of the same degree, we write $P \succ Q$ if $P(z) \geqq Q(z)$ for all $z$, and $P \not \equiv Q$ are not identical. In other words, $P>Q$ if and only if $P(z)>Q(z)$ except possibly at a finite number of points.

Case 1. First, the conditions

$$
i^{3}=j^{2} \neq 0, \quad i H-j P \succ 0,
$$

are necessary and sufficient for $P$ to have the canonical form $v\left(z^{2}+1\right)$. The normalizability conditions (5.10) can then be written as

$$
\left(i H-j P, Q^{2}\right)^{(4)}=0, \text { and } 3(T, Q)^{(2)} \succ 10 n(i H-j P) .
$$

Indeed, for $P$ in canonical form, and $Q(z)=\hat{b} z^{2}+\hat{c} z+\hat{d}$, we find

$$
\begin{gathered}
i=v^{2}, \quad j=-v^{3}, \quad i H-j P=3 v^{4}, \quad T=v^{3} z, \\
\left(i H-j P, Q^{2}\right)^{(4)}=1728 v^{4} \hat{b}^{2}, \quad(T, Q)^{(2)}=10 v^{3}(4 \hat{b} z-\hat{c}),
\end{gathered}
$$

which proves that (6.13) is equivalent to (5.10). (Note that $i H-j P$ is a quartic polynomial, i.e., transforms under the representation of weight $(4,-2)$, which happens to be constant in the given coordinates; similarly $T$ is a sextic and $(T, Q)^{(2)}$ a quartic.) In particular, since $\hat{b}$ must vanish, both $(T, Q)^{(2)}$ and $i H-j P$ are constant, so the second inequality in (6.13) says $3(T, Q)^{(2)}>10 n(i H-j P)$ except at $\infty$ (where they are both 0 ), so in a general coordinate system we need only check the inequality in (6.13) at most at two different points. A similar remark applies to $i H-j P$.

Case 2. The necessary and sufficient conditions for $P$ to have the canonical form $v\left(z^{2}-1\right)$ are

$$
i^{3}=j^{2} \neq 0, \quad i H-j P \prec 0,
$$

where, for $P$ in canonical form, $i=v^{2}, j=-v^{3}$ and $i H-j P=-3 v^{4}$. Now the problem could either be of the form in Case $2 a$ or the alternative Case $2 b$, and so we must take the two alternative sets of normalizability conditions into account simultaneously. The problem splits into two subcases, depending on whether or not $\hat{b}$ vanishes. Assume first that $\hat{b} \neq 0$. Note that, according to (5.14) or (5.18), the 
coefficient $\hat{b}$ can have either sign, depending on whether we are in Case 2a or Case $2 \mathrm{~b}$. We find, by direct computation, that if $P$ is in canonical form, then

$$
\left(i H-j P, Q^{2}\right)^{(4)}=-1728 v^{4} \hat{b}^{2} ;
$$

therefore this case is characterized by the invariant condition

$$
\left(i H-j P, Q^{2}\right)^{(4)}<0 .
$$

Next, consider the regularity conditions, which are either (5.12) or (5.17), depending on whether we are in case $2 a, \hat{b}>0$, or case $2 b, \hat{b}<0$. We can re-express these alternatives using a single condition, namely

$$
\hat{b}(\hat{b}+\hat{d})=|\hat{b}|\left(\hat{c}-n^{*} v\right),
$$

where, according to the two sub-alternatives, $n^{*}$ denotes either $n$ or $n+2$. We now express the normalizability condition (6.17) (in conjunction with (6.16)) in invariant form. We find, by direct computation, that if $P$ is in canonical form, then

$$
\begin{aligned}
\left(2 j H+i^{2} P, Q^{2}\right)^{(4)}-144 i j(Q, Q)^{(2)} & =1728 v^{5} \hat{b}(\hat{b}+\hat{d}), \\
\left(T, Q^{3}\right)^{(6)} & =259200 v^{3} \hat{b}^{2} \hat{c} .
\end{aligned}
$$

Therefore (6.17) has the following rather complicated invariant form

$$
\begin{aligned}
12 i^{2}\left(T, Q^{3}\right)^{(6)} & +1800 n^{*} i^{2}\left(i H-j P, Q^{2}\right)^{(4)} \\
& =25 \sqrt{\left(3 j P-3 i H, Q^{2}\right)^{(4)}}\left[\left(2 j H+i^{2} P, Q^{2}\right)^{(4)}-144 i j(Q, Q)^{(2)}\right],
\end{aligned}
$$

where $n^{*}=n$ or $n+2$. Note that each term in (6.18) is an (absolute) invariant. Unfortunately, no simpler formulation of the normalizability conditions seems to work!

Alternatively, suppose $\hat{b}=0$, which is characterized by the invariant condition

$$
\left(i H-j P, Q^{2}\right)^{(4)}=0 .
$$

Direct calculation of transvectants shows that the regularity conditions (5.12) now have the invariant formulation

$$
25\left[\left(P, Q^{2}\right)^{(3)}\right]^{2}(i H-j P)=864(Q, Q)^{(2)}\left[3(T, Q)^{(2)}+10 n^{*}(i H-j P)\right]^{2} .
$$

Similarly, the normalizability condition (5.16) (which is the same in both cases) has the invariant form

$$
3(T, Q)^{(2)} \prec 10 n(i H-j P) .
$$

Case 3. This is similar to Case 2, but computationally easier. First the necessary and sufficient conditions for $P$ to have the canonical form $v z^{2}$ are

$$
i^{3}=j^{2} \neq 0, \quad T=0 .
$$

The set of full normalizability conditions, which are (5.24), (5.25), or their alternatives (5.29), (5.30), are equivalent to the following three alternative possibilities. The first is $\hat{b} \hat{d}<0$, which can be written in invariant form as

$$
\left(H, Q^{2}\right)^{(4)}-48 i(Q, Q)^{(2)}>0 .
$$


The second, which is $\hat{b}=0, \hat{c}<-n v, \hat{d} \neq 0$, and third, which is $\hat{d}=0, \hat{c}>n v$, $\hat{b} \neq 0$, both have the same invariant form:

$$
\left(H, Q^{2}\right)^{(4)}=48 i(Q, Q)^{(2)}, \quad(H, Q)^{(2)}-8 i Q \not \equiv 0, \quad \frac{\left(P, Q^{2}\right)^{(3)}}{(H, Q)^{(2)}-8 i Q}<12 n .
$$

Case 4.

$$
i=j=0, \quad H \not \equiv 0,
$$

are necessary and sufficient for $P$ to have the canonical form $z$. The normalizability conditions (5.32), (5.33) can then be written as either

$$
\left(T, Q^{3}\right)^{(6)}>0, \quad 2400(Q, Q)^{(2)}\left(H, Q^{2}\right)^{(4)}-25\left[\left(P, Q^{2}\right)^{(4)}\right]^{2}=64 n^{*}\left(T, Q^{3}\right)^{(6)},
$$

or

$$
\left(T, Q^{3}\right)^{(6)}=0, \quad(P, Q)^{(2)} \succ 0, \quad 3 P(H, Q)^{(1)}-6 H(P, Q)^{(1)}=8 n^{*} H^{2},
$$

where $n^{*}$ is either $n$ or $n+2$. In the second equality in (6.27), the left-hand side is a (real) eighth degree polynomial, constant in the canonical coordinates, and hence the equality needs only be checked at three distinct points.

Case 5.

$$
i=j=T=H=0,
$$

are necessary and sufficient for $P$ to have the constant canonical form 1 . The normalizability conditions $(5.35)$ can then be written as

$$
\left(P, Q^{2}\right)^{(4)}=0, \quad \text { and }(P, Q)^{(1)} \succ 0 .
$$

Again, the first condition implies $\hat{b}=0$, so $(P, Q)^{(1)}=-4 \hat{c}$ is constant, and so the inequality needs only be checked for at most two points.

It is worth re-emphasizing that each of the previous invariant normalizability conditions can be explicitly written in terms of the coefficients of the quartic and quadratic polynomials defining the operator, and hence, using (2.8), directly in terms of the Lie algebraic coefficients $c_{a b}, c_{a}$. Apart from the discriminantal condition (6.9), the resulting expressions, however, are too unwieldy to warrant displaying here. Rather, for any given operator, a straightforward calculation based on the explicit transvectant formulas for the covariants will determine whether or not it satisfies the normalizability criteria.

\section{Singular Potentials and Normalizability}

Cases 2 and 4 also included singular quasi-exactly solvable potentials, and we now analyze these in some more detail. When the potential $V(x)$ is singular at the origin it is of the form

$$
V(x)=U(x)+\frac{\lambda(\lambda+1)}{x^{2}},
$$

with $U$ smooth and $\lambda \in \mathbb{R}$. In the canonical coordinate,

$$
\lambda= \begin{cases}\hat{d}-\frac{1}{2} n-1 & \text { Case } 2 \mathrm{a}, \\ \frac{\hat{b}+\hat{c}+\hat{d}}{2 v}-\frac{n}{2}-1, & \text { Case } 4 .\end{cases}
$$


The question is whether we can assign a physical interpretation to such a potential, and, consequently, appropriate normalizability conditions. A potential of this type, with $\lambda=l \in \mathbb{N}$ a nonnegative integer representing the total angular momentum of the particle, arises naturally in quantum mechanics when one tries to solve the Schrödinger equation for a spherically symmetric Hamiltonian by separation of variables. Indeed, suppose $\psi(x)$ is an eigenfunction for the Schrödinger operator $\mathscr{S}=-D_{x}^{2}+V(x)$ on $0<x<\infty$ with limit

$$
\lim _{x \rightarrow 0+} \psi(x)=\psi(0)=0
$$

at the origin. Let $r, \theta, \varphi$ be spherical coordinates in $\mathbb{R}^{3}$, and $\Delta$ denote the standard flat space Laplacian in $\mathbb{R}^{3}$. Given $\lambda=l \in \mathbb{N}$ and a spherical harmonic $Y_{l m}(\theta, \varphi)$, the function

$$
\Psi(r, \theta, \varphi)=\frac{\psi(r)}{r} Y_{l m}(\theta, \varphi)
$$

is an eigenfunction for the three-dimensional Schrödinger operator $\mathscr{H}=$ $-\Delta+U(r)$. Furthermore,

$$
\Psi \in L^{2}\left(\mathbb{R}^{3}\right) \text { if and only if } \psi \in L^{2}(0, \infty) .
$$

Thus, if the parameter $\lambda$ is a nonnegative integer, we can regard the Schrödinger equation for the potential $(7.1)$ on $(0, \infty)$ as the radial Schrödinger equation for the regular three-dimensional potential $U(r)$ at angular momentum $\lambda$, provided that we use (7.3) as the boundary condition at the origin.

If, however, $\lambda$ is not a nonnegative integer, we can no longer identify the singular part of $V$ with a centrifugal term, and the singularity of $V$ at the origin is unavoidable. When $\lambda \notin \mathbb{N}$, we shall therefore interpret the Schrödinger equation for (7.1) as the radial Schrödinger equation for the singular potential $V(r)$ at zero angular momentum. (Actually, by redefining the coefficient of the singular term we could obtain any angular momentum, but we would not gain anything by doing that.) The boundary condition at the origin is still (7.3), but now we have the additional problem of ascertaining whether the singularity of $V$ at the origin is "physical."

We recall some standard facts on three-dimensional spherically symmetric potentials of the form

$$
V(r)=U(r)+\frac{\gamma}{r^{2}}
$$

with $U$ smooth and $\lim _{r \rightarrow+\infty} V(r)>-\infty$.

i) If

$$
\gamma \geqq-\frac{1}{4}
$$

ii) If

then the spectrum of $\mathscr{H}$ is bounded from below, [18].

$$
-\frac{1}{4}<\gamma<\frac{3}{4}
$$

then $\mathscr{H}$ is not essentially self-adjoint on $C^{\infty}\left(\mathbb{R}^{3} \backslash\{0\}\right)$, but it has a self-adjoint extension which is physically acceptable, [4; p. 230]. 
iii) If

$$
\gamma \geqq \frac{3}{4}
$$

then $\mathscr{H}$ is essentially self-adjoint on $C^{\infty}\left(\mathbb{R}^{3} \backslash\{0\}\right),[18]$.

Thus the potential (7.4) is physical at least for $\gamma>-\frac{1}{4}$. It is not so clear what happens for the borderline value $\gamma=-\frac{1}{4}$. The asymptotics of the eigenfunctions of the potential (7.4) at the origin demonstrate that the average value of the potential energy (and hence also the kinetic energy) over an eigenfunction of (7.4) is necessarily infinite, which is problematic for physical applications, cf. [4; p. 228]. The same argument will show that this doesn't happen in cases ii) and iii). In fact, in case iii) there is only one square integrable eigenfunction of $\mathscr{H}$ near the origin (limit-point case), for which the average potential and kinetic energies are finite. In case ii), there are two square integrable eigenfunctions of $\mathscr{H}$ near the origin (limit-circle case), but the average potential and kinetic energy is finite for exactly one of these eigenfunctions. In fact, the condition of possessing a finite average potential and kinetic energy is the extra boundary condition one has to impose in this case to single out the "physical" self-adjoint extension of $\mathscr{H}$ from the infinitely many mathematically possible self-adjoint extensions.

Summarizing, the (radial) potential (7.4) is physically meaningful provided that

$$
\gamma>-\frac{1}{4}
$$

which implies that all values of $\lambda$ are acceptable except for $\lambda=-\frac{1}{2}$. Notice, however, the two different physical interpretations for the eigenvalue problem of (7.1) if $\lambda$ is a nonnegative integer. The boundary condition in the singular case is given by (7.3) for all values of $\lambda$.

Applying these remarks to our problem, first consider the singular potentials of type (5.31), assuming that the regularity condition (5.32) is not satisfied. The algebraic eigenfunctions for $x>0$ are given by

$$
\psi_{k}(x)=\left(\frac{x}{2}\right)^{\hat{d}-\frac{n}{2}} \exp \left[\frac{\hat{b}}{64} x^{4}+\frac{\hat{c}}{8} x^{2}\right] \chi_{k}\left(\frac{x^{2}}{4}\right)
$$

The boundary condition $\psi(0)=0$ implies that $\hat{d}>\frac{1}{2} n$, and the full normalizability conditions in this case are

$$
\hat{d}>\frac{n}{2}, \quad \text { and } \quad \hat{d} \neq \frac{n+1}{2} .
$$

Direct calculation of transvectants shows that the first condition has the invariant formulation

$$
\left(T, Q^{3}\right)^{(6)}>0: \quad 64 n\left(T, Q^{3}\right)^{(6)}+25\left[\left(P, Q^{2}\right)^{(4)}\right]^{2}<2400(Q, Q)^{(2)}\left(H, Q^{2}\right)^{(4)},
$$

or

$$
\left(T, Q^{3}\right)^{(6)}=0: \quad 8 n H^{2}+3 P(H, Q)^{(1)}>6 H(P, Q)^{(1)}
$$


The second condition in (7.9) is similar:

$$
\left(T, Q^{3}\right)^{(6)}>0: \quad 64(n+1)\left(T, Q^{3}\right)^{(6)}+25\left[\left(P, Q^{2}\right)^{(4)}\right]^{2} \neq 2400(Q, Q)^{(2)}\left(H, Q^{2}\right)^{(4)},
$$

or

$$
\left(T, Q^{3}\right)^{(6)}=0: \quad 8(n+1) H^{2}+3 P(H, Q)^{(1)} \neq 6 H(P, Q)^{(1)} .
$$

Again, remember that the physical interpretation (and even the domain of the $x$ coordinate and the particular boundary conditions) of the eigenvalue problem for $\mathscr{S}$ changes as $\hat{d}$ varies on $\left[\frac{1}{2} n, \infty\right) \backslash\left\{\frac{1}{2}(n+1)\right\}$. In particular, the values of $\hat{d}$ for which the potential describes a three-dimensional anharmonic oscillator with angular momentum $l$ are given by

$$
\hat{d}=\frac{n}{2}+l+1, \quad l \in \mathbb{N} .
$$

For $l=0$, i.e., for $\hat{d}=\frac{1}{2} n+1, V$ is actually regular, but we have seen before that the algebraic method gives odd eigenfunctions. Hence these eigenfunctions automatically satisfy the boundary condition $\psi(0)=0$, and their restrictions to $(0, \infty)$ are therefore solutions of the radial Schrödinger equation with potential $A x^{6}+B x^{4}+C x^{2}$ and zero angular momentum. Thus, for this value of $\hat{d}$ we have two different (mathematically equivalent) physical interpretations. When $\hat{d}$ is neither of the form (5.32) nor (7.14) then we are still dealing with a radial Schrödinger equation, but the potential is singular at the origin and we take the angular momentum equal to zero.

Similarly, in Case $2 \mathrm{a}$, the eigenfunctions $\psi_{k,+}(x), x>0$, are given explicitly by (5.13). The boundary condition (7.3) and full normalizability requires that $\hat{b}+\hat{c}+\hat{d}>n v$ and either $\hat{b}<0$, or $\hat{b}=0, \hat{c}<-n v$. The analogous conditions for Case $2 \mathrm{~b}$ are $-\hat{b}+\hat{c}-\hat{d}>n v$ and either $\hat{b}>0$, or $\hat{b}=0, \hat{c}<-n v$. Therefore, the range of parameters for which all the algebraic eigenfunctions in one of the two subcases are normalizable is given by either

$$
\hat{b}=0, \quad \hat{c}<-n v, \quad|\hat{d}|>n v-\hat{c},
$$

or

$$
\hat{b}(\hat{b}+\hat{d})<|\hat{b}|(\hat{c}-n v) .
$$

Direct calculation of transvectants shows that these have the invariant formulation

$$
\begin{aligned}
& \left(i H-j P, Q^{2}\right)^{(4)}=0, \\
& 3(T, Q)^{(2)} \prec 10 n(i H-j P), \\
& 25\left[\left(P, Q^{2}\right)^{(3)}\right]^{2}(i H-j P)-864(Q, Q)^{(2)}\left[3(T, Q)^{(2)}+10 n(i H-j P)\right]^{2}<0
\end{aligned}
$$

or

$$
\begin{aligned}
& 12 i^{2}\left(T, Q^{3}\right)^{(6)}+1800 n i^{2}\left(i H-j P, Q^{2}\right)^{(4)} \\
& \quad-25 \sqrt{\left(3 j P-3 i H, Q^{2}\right)^{(4)}}\left[\left(2 j H+i^{2} P, Q^{2}\right)^{(4)}-144 i j(Q, Q)^{(2)}\right]>0,
\end{aligned}
$$

respectively. Finally the case $\lambda=-\frac{1}{2}$ that has a questionable physical interpretation can be expressed in invariant form as either (6.16), (6.18), or (6.19), (6.20), the second equation in each pair having $n^{*}=n+1$. 
Acknowledgements. We would like to thank A. Turbiner for bringing this problem to our attention and for helpful comments, and A. Galindo for advice on the physical interpretation of singular potentials.

\section{References}

1. Alhassid, Y., Engel, J., Wu, J.: Algebraic approach to the scattering matrix. Phys. Rev. Lett. 53, 17-20 (1984)

2. Alhassid, Y., Gürsey, F., Iachello, F.: Group theory approach to scattering. Ann. Phys. 148, 346-380 (1983)

3. Alhassid, Y., Gürsey, F., Iachello, F.: Group theory approach to scattering. II. The euclidean connection. Ann. Phys. 167, 181-200 (1986)

4. Galindo, A., Pascual, P.: Quantum Mechanics I. Berlin, Heidelberg, New York: Springer 1990

5. González-López, A., Kamran, N., Olver, P.J.: Lie algebras of differential operators in two complex variables. Am. J. Math., to appear

6. González-López, A., Kamran, N., Olver, P.J.: Quasi-exactly solvable Lie algebras of first order differential operators in two complex variables. J. Phys. A 24, 3995-4008 (1991)

7. González-López, A., Kamran, N., Olver, P.J.: New quasi-exactly solvable Hamiltonians in two dimensions. Preprint, Univ. of Minnesota 1992

8. Gorsky, A.: Relationship between exactly solvable and quasi-exactly solvable versions of quantum mechanics with conformal block equations in 2D theories. JETP Lett. 54, 289-292 (1991)

9. Grace, J.H., Young, A.: The Algebra of Invariants. Cambridge: Cambridge Univ. Press 1903

10. Gradshteyn, I.S., Ryzhik, I.W.: Table of Integrals, Series and Products. New York: Academic Press 1965

11. Gurevich, G.B.: Foundations of the Theory of Algebraic Invariants. Groningen, Holland: P. Noordhoff 1964

12. Kamran, N., Olver, P.J.: Lie algebras of differential operators and Lie-algebraic potentials. J. Math. Anal. Appl. 145, 342-356 (1990)

13. Landau, L.D., Lifshitz, E.M.: Quantum Mechanics (Non-relativistic Theory). Course of Theoretical Physics, vol. 3. New York: Pergamon Press 1977

14. Levine, R.D.: Lie algebraic approach to molecular strucure and dynamics. In: Mathematical Frontiers in Computational Chemical Physics, D.G. Truhlar (ed.) IMA Volumes in Mathematics and its Applications, vol. 15. Berlin Heidelberg, New York: Springer 1988, pp. 245-261

15. Littlejohn, L.L.: On the classification of differential equations having orthogonal polynomial solutions. Ann. di Mat. 138, 35-53 (1984)

16. Miller, W., Jr.: Lie Theory and Special Functions. New York: Academic Press 1968

17. Morozov, A.Y., Perelomov, A.M., Rosly, A.A., Shifman, M.A., Turbiner, A.V.: Quasi-exactly solvable quantal problems: one-dimensional analogue of rational conformal field theories. Int. J. Mod. Phys. 5, 803-832 (1990)

18. Reed, M., Simon, B.: Functional Analysis, vol. 2. New York: Academic Press 1975

19. Shifman, M.A.: New findings in quantum mechanics (partial algebraization of the spectral problem). Int. J. Mod. Phys. A 126, 2897-2952 (1989)

20. Shifman, M.A.: Quasi-exactly solvable spectral problems and conformal field theory. preprint, Theoretical Physics Inst., Univ. of Minnesota 1992

21. Shitman, M.A., Turbiner, A.V.: Quantal problems with partial algebraization of the spectrum. Commun. Math. Phys. 126, 347-365 (1989)

22. Turbiner, A.V.: Quasi-exactly solvable problems and sl(2) algebra. Commun. Math. Phys. 118, 467-474 (1988)

23. Turbiner, A.V.: Lie algebras and polynomials in one variable. J. Phys. A 25, L1087-L1093 (1992)

24. Ushveridze, A.G.: Quasi-exactly solvable models in quantum mechanics. Sov. J. Part. Nucl. 20, 504-528 (1989)

25. Wilczynski, E.J.: Projective Differential Geometry of Curves and Ruled Surfaces. Leipzig: B.G. Teubner 1906 